\title{
THE CAUCHY INTEGRAL FOR FUNCTIONS OF SEVERAL COMPLEX VARIABLES
}

\author{
BY WALTER KOPPELMAN ${ }^{1}$
}

Communicated by Murray Gerstenhaber, December 2, 1966

This note contains a new contribution to the already vast literature on the topic under discussion. While we were led to the present considerations through a remark in a paper of Bochner [4] and originally arrived at our results more or less independently, we found, upon turning to the literature of the intervening twenty years, that our work was quite closely related to papers by Weil, Sommer, Arens, Leray, Norguet, Waelbroeck, Gleason, and Aľzenberg [11], [9], [3], [6], [7], [10], [5], [1]. Nevertheless, our approach offers some clarification of the existing theory and, indeed, we demonstrate this assertion by discussing a few applications. The subjects outlined here constitute a portion of material that will appear in forthcoming publications.

1. Cauchy-Fantappiè forms. Let $\zeta \in \boldsymbol{C}^{n}$. We shall be interested in certain mappings of neighborhoods of $\zeta$ into $\boldsymbol{C}^{n}$. Let us, in particular, consider two such mappings, $\psi$ and $f$, where $\psi$ is of class $C^{\infty}$ and $f$ is holomorphic. Writing $\psi=\left(\psi_{1}, \cdots, \psi_{n}\right)$ and $f=\left(f_{1}, \cdots, f_{n}\right)$, we set $\langle\psi, f\rangle=\psi_{1} f_{1}+\cdots+\psi_{n} f_{n}$. As usual, we decompose the operation $d$ of exterior differentiation into its two components $\partial, \bar{\partial}$, of bidegree $(1,0)$ and $(0,1)$ respectively, so that $d=\partial+\bar{\partial}$. Next, we introduce the two differential forms $\omega(f)=d f_{1} \wedge \cdots \wedge d f_{n}$ and

$$
\omega^{\prime}(\psi)=\sum_{j=1}^{n}(-1)^{j-1} \psi_{j} \bar{\partial} \psi_{1} \wedge \cdots \wedge \bar{\partial} \psi_{j-1} \wedge \bar{\partial} \psi_{j+1} \wedge \cdots \wedge \bar{\partial} \psi_{n}
$$

If $\langle\psi, f\rangle(\zeta) \neq 0$, then the differential form

$$
(-1)^{n(n-1) / 2}(n-1) !(2 \pi i\langle\psi, f\rangle)^{-n} \omega^{\prime}(\psi) \wedge \omega(f)
$$

will be called a Cauchy-Fantappiè (C-F) form of type $f$ at $\zeta$. When $\psi=\bar{f}$, we shall speak of the Bochner-Martinelli (B-M) form of type $f$. The fundamental structure of $\mathrm{C}-\mathrm{F}$ forms follows from

TheOREM 1.1. (i) $\bar{\partial}\langle\psi, f\rangle^{-n} \omega^{\prime}(\psi)=0$.

(ii) If the functions $\left\langle\psi^{(j)}, f\right\rangle, j=1,2$, do not vanish on an open neighborhood $V_{\zeta}$ of $\zeta$, then there exists a differential form $\beta$ of bidegree $(0, n-2)$ on $V_{\zeta}$, such that

1 The author gratefully acknowledges support by the National Science Foundation under grant GP-6102. 


$$
\left\langle\psi^{(1)}, f\right\rangle^{-n} \omega^{\prime}\left(\psi^{(1)}\right)-\left\langle\psi^{(2)}, f\right\rangle^{-n} \omega^{\prime}\left(\psi^{(2)}\right)=\bar{\partial} \beta .
$$

In particular, the C-F forms of type $f$ are $\bar{\partial}$-closed (hence closed) and the difference of two such forms is $\bar{\partial}$-exact (hence exact). The proof of Theorem 1.1 is best obtained by imbedding the family of forms in the larger family

$$
D\left(\left\langle\psi^{(1)}, f\right\rangle^{-1} \psi^{(1)}, \bar{\partial}\left(\left\langle\psi^{(2)}, f\right\rangle^{-1} \psi^{(2)}\right), \cdots, \bar{\partial}\left(\left\langle\psi^{(n)}, f\right\rangle^{-1} \psi^{(n)}\right)\right),
$$

where $D$ is a determinant with the indicated column vectors, but where exterior multiplication is used in place of ordinary multiplication.

Proposition 1.2. The expression (1.3) is independent of the choice of the mapping $\psi^{(1)}$.

This immediately implies that the members of the family (1.3) are $\bar{\partial}$-closed and that the difference of two members is $\bar{\partial}$-exact.

Proposition 1.3. $(n-1) !\langle\psi, f\rangle^{-n} \omega^{\prime}(\psi)=D\left(\langle\psi, f\rangle^{-1} \psi, \bar{\partial}\left(\langle\psi, f\rangle^{-1} \psi\right)\right.$, $\left.\cdots, \bar{\partial}\left(\langle\psi, f\rangle^{-1} \psi\right)\right)$.

Thus Theorem 1.1 is proved.

2. The Cauchy integral formula. Suppose that $g$ is holomorphic in a domain $D \subset C^{n}$ containing the point $z$, and that $\gamma$ is a cycle ${ }^{2}$ in $D$ of degree 1 with respect to the point $z$. With $f \equiv \zeta-z$ and $\langle\psi, \zeta-z\rangle \neq 0$ at all points $\zeta$ of $\gamma$, we multiply the form (1.2) by $g$ and integrate over $\gamma$. Now, in view of Theorem 1.1 and Stokes' theorem, we may replace $\psi$ by $\bar{f}$ and $\gamma$ by a sphere with center $z$. However, now the integral simply reduces to the mean value of $g$ over that sphere, so that our integral is equal to $g(z)$.

3. Residues and local properties of holomorphic mappings. Let us suppose that $D$ is a domain with $0 \in D \subset C^{n}$ and $f: D \rightarrow C^{n}$ is a holomorphic mapping with $f(0)=0$. If all components of $f$ have power series expansions of degree 1 about 0 , we may write $f=A z$ near 0 , where $A$ is a matrix of holomorphic functions, such that $\operatorname{det} A(0)$ $=J(f)(0)$, the complex Jacobian of the mapping $f$ at 0 . We now observe that $\langle\psi, f\rangle=\langle\psi, A z\rangle=\left\langle{ }^{t} A \psi, z\right\rangle$, and that $\omega^{\prime}\left({ }^{t} A \psi\right)=(\operatorname{det} A) \omega^{\prime}(\psi)$. In particular, when $\operatorname{det} A(0) \neq 0$, we have

$$
\langle\psi, f\rangle^{-n} \omega^{\prime}(\psi) \wedge \omega(f)=(\operatorname{det} A)^{-1} J(f)\left\langle{ }^{t} A \psi, z\right\rangle^{-n} \omega^{\prime}\left({ }^{t} A \psi\right) \wedge \omega(z)
$$

near 0 . This identity, together with Theorem 1.1 and the results of $\S 2$, yields

2 More precisely, a compact bounding $(2 n-1)$-cycle. 
Proposition 3.1. Suppose that $D$ is a domain with $0 \in D \subset C^{n}$, and $f: D \rightarrow C^{n}$ is a holomorphic mapping such that $f^{-1}(\{0\})=\{0\}$ and $J(f)(0) \neq 0$; then, if $g$ is a function holomorphic in $D, \gamma$ is a cycle in $D$ of degree 1 with respect to 0 , and $\langle\psi, f\rangle \neq 0$ at all points of $\gamma$,

$$
(-1)^{n(n-1) / 2}(n-1) !(2 \pi i)^{-n} \int_{\gamma} g\langle\psi, f\rangle^{-n} \omega^{\prime}(\psi) \wedge \omega(f)=g(0) .
$$

We stress that we have arrived at this result without employing the implicit function theorem. Consequently, we can obtain a new proof of this theorem by applying the ideas used for functions of one complex variable. Furthermore, we may also use such a method together with Sard's theorem, to establish the classical theorem that if $f$ is 1-1 on $D$, then $f$ is an open mapping with a holomorphic inverse.

4. Problems of analytic continuation. We shall be interested in complex manifolds $M$ of dimension $n \geqq 2$, which satisfy the condition

(*) For each $p \in M$, there exists a holomorphic mapping $f_{p}: M \rightarrow \boldsymbol{C}^{n}$, which is nonsingular at $p$, such that $f_{p}^{-1}(\{0\})=\{p\}$.

Notation. $\Omega^{\nu}=$ sheaf of germs of holomorphic forms of degree $\nu, H_{*}$ refers to cohomology with compact supports.

Following ideas of Bochner [4], we can now prove

TheOREM 4.1. Let $M$ be a paracompact connected complex manifold of dimension $n \geqq 2$, which satisfies condition (*). Suppose that there exists an increasing sequence $\left\{D_{j}\right\}$ of relatively compact open sets in $M$, such that $M=\mathrm{U}_{j} D_{j}$ and $H^{n-1}\left(D_{j}, \Omega^{n}\right)=0$ for each $j$; then $H_{*}^{1}\left(M, \Omega^{0}\right)=0$.

The proof employs C-F forms of type $f_{p}$ together with a local version of an extension problem for $\bar{\partial}$-closed forms of type $(n, n-1)$ due to Andreotti and Vesentini [2]. The theorem implies, in particular, that $H_{2 n-1}^{*}(M, Z)=0$ and that if $D$ is a relatively compact domain in $M$ with a connected boundary $\partial D$, then any function $g$, holomorphic on $\partial D$, has a single-valued analytic extension to all of $D$ (Hartogs' theorem). When $\partial D$ is smooth, the last statement even holds when $g$ is Hölder continuous and satisfies the tangential Cauchy-Riemann equations in the sense of the theory of distributions. Theorem 4.1 is, of course, closely related to a special case of Serre's duality theorem [8]. If $M$ is a Stein manifold, the hypotheses of Theorem 4.1 are fulfilled.

If we apply Cartan's Theorem B to our integration theory, we obtain the following interesting result.

TheORem 4.2. Let $M$ be a Stein manifold of dimension $n \geqq 2, K a$ compact subset of $M, \partial K$ the boundary of $K, L$ a closed subset of $\partial K$, 
and $p \in K$. If there exists a function $h$, holomorphic on $K$, such that $|h(p)|>\max _{q \in L}|h(q)|$, then for every open set $V$, such that $\partial K-L$ $\subset V \subset M-\{p\}$, the envelope of holomorphy $E(V)$ must contain a point $\tilde{p}$ which projects onto $p$.

5. Banach algebras. We shall only be concerned with unitary commutative Banach algebras $B$ over the field $C$ of complex numbers. The spectrum $\sigma(B)$ is given the Gelfand topology. If $f_{1}, \cdots, f_{n} \in B$, then the mapping $f: \sigma(B) \rightarrow \boldsymbol{C}^{n}$ defined by $f(\chi)=\left(\chi\left(f_{1}\right), \cdots, \chi\left(f_{n}\right)\right)$, $\chi \in \sigma(B)$ is continuous. The compact subset $f(\sigma(B))$ of $\boldsymbol{C}^{n}$ is called the joint spectrum of $f_{1}, \cdots, f_{n}$. A point $z=\left(z_{1}, \cdots, z_{n}\right) \in C^{n}$ lies in the complement $f(\sigma(B))^{\prime}$ of the joint spectrum if and only if the ideal $\left(z_{1}-f_{1}, \cdots, z_{n}-f_{n}\right)$ is the unit ideal of $B$. In that case, there exist elements $a_{1}, \cdots, a_{n} \in B$, such that $a_{1}\left(z_{1}-f_{1}\right)+\cdots+a_{n}\left(z_{n}-f_{n}\right)=1$. If $\zeta \in C^{n}$ is close to $z$, then the element $a_{1}\left(\zeta_{1}-f_{1}\right)+\cdots+a_{n}\left(\zeta_{n}-f_{n}\right)$ is close to 1 and thus has an inverse, $b(\zeta)$, which depends holomorphically on $\zeta$. Setting $b(\zeta) a_{j}=\phi_{j}(\zeta)$, we have $\phi_{1}(\zeta)\left(\zeta_{1}-f_{1}\right)+\cdots$ $+\phi_{n}(\zeta)\left(\zeta_{n}-f_{n}\right) \equiv 1$ for $\zeta$ near $z$. By using these local expressions together with a partition of unity, we can construct a $C^{\infty}$ mapping $\psi: f(\sigma(b))^{\prime} \rightarrow B^{n}$, such that $\langle\psi(\zeta), \zeta-f\rangle=\psi_{1}(\zeta)\left(\zeta_{1}-f_{1}\right)+\ldots$ $+\psi_{n}(\zeta)\left(\zeta_{n}-f_{n}\right) \equiv 1$ on $f(\sigma(B))^{\prime}$. We can therefore construct B-valued C-F forms, defined on $f(\sigma(B))^{\prime}$, for which the propositions of $\$ 1$ are valid. As an example of an application, we formulate a type of Cousin problem.

Theorem 5.1. Suppose that $B, B_{1}, B_{2}$ are Banach algebras with continuous homomorphisms $r_{j}: B \rightarrow B_{j}$. Let $f_{1}, \cdots, f_{n} \in B$ and define $f_{1}^{(j)}, \cdots, f_{n}^{(j)}$ by $f_{k}^{(j)}=r_{j} f_{k}$. Suppose that $g$ is a given function, holomorphic on the compact set $K=f^{(1)}\left(\sigma\left(B_{1}\right)\right) \cap f^{(2)}\left(\sigma\left(B_{2}\right)\right)$. A ssume that there exists $a(2 n-1)$-cycle $\gamma$ in the region of definition of $g$ with the following properties:

(i) $\gamma$ is of degree 1 about each point of $K$;

(ii) for each $z \in K$, the Cauchy integral of $g$ over $\gamma$ yields the value $g(z)$;

(iii) there exist open sets $U, U_{1}, U_{2}$, with $U_{j} \subset f^{(j)}\left(\sigma\left(B_{j}\right)\right)^{\prime}, \quad U$ $C f^{(1)}\left(\sigma\left(B_{1}\right)\right)^{\prime} \cap f^{(2)}\left(\sigma\left(B_{2}\right)\right)^{\prime} \cap f(\sigma(b))^{\prime}$, and $U_{1} \cap U_{2}=\varnothing$, such that $\gamma \subset U_{1} \cup U_{2} \cup U$; then there exist elements $G_{1} \in B_{1}$ and $G_{2} \in B_{2}$, such that whenever $\chi_{1} \in \sigma\left(B_{1}\right), \quad \chi_{2} \in \sigma\left(B_{2}\right), \quad$ and $\quad \chi_{1} r_{1}=\chi_{2} r_{2}, \quad \chi_{1}\left(G_{1}\right)+\chi_{2}\left(G_{2}\right)$ $=g\left(f^{(1)}\left(\chi_{1}\right)\right)=g\left(f^{(2)}\left(\chi_{2}\right)\right)$.

\section{BIBLIOGRAPHY}

1. L. A. Afzzenberg, Integral representations of holomorphic functions of several complex variables, Soviet Math. Dokl. 5 (1964), 307-311.

2. A. Andreotti and E. Vesentini, Carleman estimates for the Laplace-Beltrami 
equation on complex manifolds, Inst. Hautes Etudes Sci. Publ. Math. No. 25 (1965) $81-130$.

3. R. Arens, Cauchy integral for functions of several variables, Tôhoku Math. J. 8 (1956), 268-272.

4. S. Bochner, Analytic and meromorphic continuation by means of Green's formula, Ann. of Math. 44 (1943), 652-673.

5. A. M. Gleason, The Cauchy-Weil theorem, J. Math. Mech. 12 (1963), 429-444.

6. J. Leray, Le calcul differrentiel et intêgral sur une variêté analytique complexe (Problème de Cauchy III), Bull. Soc. Math. France 87 (1959), 81-180.

7. F. Norguet, Problemes sur les formes differentielles et les courants, Ann. Inst. Fourier Grenoble 11 (1961), 1-82.

8. J.-P. Serre, Un thêorème de dualite, Comment. Math. Helv. 29 (1955), 9-26.

9. F. Sommer, Über die Integralformeln in der Funktionentheorie mehrerer kom plexer Veränderlichen, Math. Ann. 125 (1952), 172-182.

10. L. Waelbroeck, Etude spectrale des algèbres completes, Acad. Roy. Belg. C1. Sci. Mém. Coll. in $-8^{\circ}$ (2) 31 (1960), no. 7.

11. A. Weil, L'intégrale de Cauchy et les fonctions de plusieurs variables, Math. Ann. 111 (1935), 178-182.

The Institute for Advanced Study and The University of Pennsylvania 